\title{
Per- and polyfluoroalkyl substances (PFASs) in Chinese drinking water: risk assessment and geographical distribution
}

\author{
Liquan Liu' ${ }^{1}$ Yingxi Qu' ${ }^{1}$ Jun Huang ${ }^{1 *}$ (I) and Roland Weber ${ }^{2}$
}

\begin{abstract}
Background: In recent years, the widespread presence of perfluoroalkyl and polyfluoroalkyl substances (PFASs) in the environment and the related exposures and adverse health effects has received increasing attention. However, PFASs are not routinely monitored in drinking water in many parts of the world, including China. PFAS data are mainly generated by research studies. This paper provides an overview of the available research studies on PFASs in Chinese drinking water to better understand the current status of PFAS contamination and the potential for exposure.

Results: The available studies provided PFAS data from 526 drinking water samples across 66 cities in China with a total of approximately 452 million inhabitants. We mapped the risk distribution associated with PFAS-contaminated drinking water in China by comparing the measured levels with recent international guidelines. The PFAS concentrations reported in more than $20 \%$ of the studied cities, likely affecting 98.5 million people, were above the maximum contaminant level issued by Vermont in 2019. Furthermore, we also investigated the human exposure to PFASs in drinking water by estimating total daily intakes based on Exposure Factors Handbook of Chinese Population. This study revealed that East China and the Southwest regions posed a relatively higher risk to the Chinese population and some cities in the Yangtze River basin such as Zigong, Jiujiang, Lianyungang and a considerable share of other cities have exceeded the health-based guidelines issued by EU and US agencies.

Conclusion: Drinking water in many cities and regions in China is contaminated with PFASs at levels of concern. PFAS elimination of PFASs from drinking water in contaminated cities and affected regions in China is urgently needed. PFAS releases from industries and other sources need better control and reduction. Further monitoring in remote Chinese regions is needed to overcome the knowledge gaps for a more comprehensive understanding of population exposure. The current risk assessment of PFASs in China should be re-evaluated considering the most recent toxicological studies, to clarify if the guidelines need to be lowered as recently done in Europe and the United States. This is necessary to have the best national base for risk assessment and a science-based driver for countermeasures.
\end{abstract}

Keywords: PFASs, Drinking water, Risk assessment, Human exposure, Total daily intake, Distributions

\footnotetext{
*Correspondence: huangjun@mail.tsinghua.edu.cn

${ }^{1}$ State Key Joint Laboratory of Environmental Simulation and Pollution Control (SKLESPC), Beijing Key Laboratory for Emerging Organic Contaminants Control (BKLEOC), Beijing Laboratory for Environmental Frontier Technologies (BLEFT), School of Environment, Tsinghua University, Beijing 100084, China

Full list of author information is available at the end of the article
}

\section{Background}

Perfluoroalkyl and polyfluoroalkyl substances (PFASs) have been extensively manufactured and consumed worldwide since the 1950s, and since then, their use has increased dramatically [1]. They are characterized by their amphiphilic properties, making them useful for various applications such as aqueous film-forming foams, semiconductors, electronics, stain-resistant coatings, oilrepellents, and additives to industrial products [2].

\section{Springer Open}

(c) The Author(s) 2021. This article is licensed under a Creative Commons Attribution 4.0 International License, which permits use, sharing, adaptation, distribution and reproduction in any medium or format, as long as you give appropriate credit to the original author(s) and the source, provide a link to the Creative Commons licence, and indicate if changes were made. The images or other third party material in this article are included in the article's Creative Commons licence, unless indicated otherwise in a credit line to the material. If material is not included in the article's Creative Commons licence and your intended use is not permitted by statutory regulation or exceeds the permitted use, you will need to obtain permission directly from the copyright holder. To view a copy of this licence, visit http://creativeco mmons.org/licenses/by/4.0/. 
The massive production and application of PFASs combined with their very high persistence and mobility have resulted in their ubiquitous presence in aquatic environments, sediments, air, biota and humans [3-7]. Therefore, they are recognized as environmental contaminants of high and emerging concern [8]. In recent years, there has been growing concern regarding the presence of PFASs in drinking water due to their potential for toxic effects and direct exposure to humans [9, 10]. Animal toxicity studies found an association between exposure to perfluorooctanoic acid (PFOA) and adverse health effects on fertility, pregnancy and many organs and systems [11, 12]. Epidemiological studies have found a connection between PFASs (especially perfluorooctanesulfonic acid (PFOS) and PFOA) exposure and health issues, including an increase in cholesterol and liver enzymes, increased incidence of testicular and kidney cancer, reduced fertility and fecundity, immune suppression and thyroid disorders, among others $[13,14]$. PFOS and PFOA were listed in Annex B and Annex A of the Stockholm Convention as Persistent Organic Pollutants (POPs) in 2009 and 2019, respectively, with a range of exemptions allowing further use $[15,16]$. Although banned by an increasing number of countries, PFOS and PFOA have been frequently detected in drinking water around the world in recent years, such as in the U.S, Japan, Ghana, Turkey, Australia and China [3, 17-21].

To control and reduce human risk from PFAS exposure, the levels of PFASs in drinking water are being increasingly regulated in many countries [22]. In 2002, the first guideline was issued in the US state of West Virginia, which set a maximum level of 150,000 ng/L PFOA in drinking water polluted by a DuPont facility; this was recently covered in a documentary film [23]. The manufacturing activities of $3 \mathrm{M}$ have also resulted in contamination with PFOA and PFOS, with concentrations in groundwater of up to 42,000 and $2700 \mathrm{ng} / \mathrm{L}$, respectively [24]. These exceeded the then groundwater criteria of 7000 and $1000 \mathrm{ng} / \mathrm{L}$ and were much higher than the 2009 drinking water criteria of 500 and $300 \mathrm{ng} / \mathrm{L}$ set by the Agency for Toxic Substances and Disease Registry (ATSDR) of the US Department of Health and Human Services 2009 [24]. In 2016, the US Environmental Protection Agency published a non-enforceable $70 \mathrm{ng} / \mathrm{L}$ health advisory (HA) for the sum of PFOA and PFOS, intended to provide protection from a lifetime of exposure from drinking water [14]. In recent years, many US states have developed their own non-enforceable HAs and enforceable maximum contaminant levels (MCLs), some more stringent than the federal guidelines. Until now, six US states have set MCLs: New Jersey, Vermont, Michigan, New York, New Hampshire and Massachusetts have set MCLs (see Additional file 1: Table S1). For example, Vermont set an MCL of $20 \mathrm{ng} / \mathrm{L}$ for PFOA, PFOS, PFHxS, PFHpA, and PFNA individually or combined in 2019 [25]. Grandjean and Budtz-Jørgensen recommended a health guideline of $1 \mathrm{ng} / \mathrm{L}$ for PFOA based on the reduced effectiveness of vaccines and impact on mammary gland development [26]. In addition, Denmark, Canada, Sweden, Australia, Germany and Italy have also proposed their own non-enforceable or regulatory guidelines for PFAS in drinking water since 2006, becoming lower and lower as emerging health studies found effects at lower levels, similar to the development in the U.S as summarized in Additional file 1: Table S1.

Given that certain PFASs, in particular PFOS and its precursors, have been gradually phased out in North America and Europe since 2002, some Asian countries, especially China, have possibly become the main manufacturers of PFOS and PFOA [27, 28]. Following a rapid increase in PFAS production and usage in China over the past 30 years, China is now one of the largest manufacturers and consumers of PFASs in the world [27]. The widespread and increasing production have resulted in an increasing risk of PFAS environmental releases and associated ground- and surface water pollution, and finally drinking water contamination in China. Due to inefficient removal in traditional drinking water treatment plants, PFASs in drinking water can pose a high risk for human exposure [29]. Extremely high levels of PFOA and PFOS were found in drinking water in several Chinese cities in the Yangtze River basin such as Zigong, Lianyungang, Shanghai, etc. [30-32]. However, there are currently no guidelines for PFASs in drinking water in China [33]. In 2019, Zhang et al. first suggested health advisory values of $85 \mathrm{ng} / \mathrm{L}$ for PFOA and $47 \mathrm{ng} / \mathrm{L}$ for PFOS in China based on analyzing the relationship between blood PFAS concentration and drinking water exposure [31]. Since PFASs are not included in routine monitoring of drinking water quality in China, the main source of data are the research studies conducted in a range of cities and regions in China. These PFAS monitoring activities have revealed different pollution levels of PFASs in a range of cities. The PFAS levels are associated with pollution source, economic activities and factor, population density and development of water treatment [34]. In addition, China has not yet conducted a national census-like PFAS monitoring in drinking water. Thus, the national distribution of drinking water PFAS contamination in China has not yet been assessed. Moreover, to the best of our knowledge, there are no reports reviewing the national status of PFAS concentrations in drinking water and analyzing the associated human health risk for China with health risk based exposure limit values. Only a review on PFAS levels in environmental matrices in China has been compiled [27]. Therefore, this study aims 
to provide a comprehensive overview of PFASs in drinking water across China from the published peer-reviewed literatures and compare the measured levels with available guidelines.

The objectives of the present study are: (1) to review the occurrence of PFASs in Chinese drinking water by screening the published literature; (2) to assess the human health risk from the contamination of drinking water with PFASs by comparing with existing guideline levels; and (3) to evaluate the potential human PFAS exposure risk from drinking water by calculating total daily intake values using Chinese population exposure parameters. We have also made efforts to reveal the major contamination source, and suggest possible risk control measures and future research needs.

\section{Materials and methods \\ Data acquisition}

We surveyed the literature on PFAS monitoring data in Chinese drinking water. The studies were extracted from Google Scholar, Science Direct, Scopus, Web of Science and the CNKI databases using the following keywords: PFASs, PFCs, PFOA, PFOS, drinking water or tap water, and China. Publications in English and Chinese were both included in this study, as well as all types of analytical methods, to better grasp the contamination status of drinking water in China. We reviewed and extracted data from a total of approx. 30 peer-reviewed publications related to PFAS monitoring of drinking water in 66 cities in China, which are listed in the supplemental material. Some of the papers reviewed did not include their data in the text or tables, so the data were extracted using GetData Graph Digitizer software, which can extract values from figures by numerical algorithm. Some of the data were not used, since we could not obtain a detailed geographical location corresponding to the PFAS concentration. All the data points are shown in Additional file 1: Table S6.

\section{Data treatment}

The arithmetic weighted average concentrations of each PFAS in the same city were calculated based on the values reported in different studies. If a substance was not detected (ND), its concentration was regarded as zero. When the reported PFAS concentrations were below the limit of quantification (LOQ), we used half of the LOQ values in subsequent calculation. Statistical analysis was conducted with the IBM SPSS Statistics software. Kruskal-Wallis tests were performed to determine the significance of differences between regional groups, using a $P$ value $<0.05$ to indicate statistical significance for all tests.

\section{Risk assessment}

To evaluate the human health risk associated with exposure to PFAS-contaminated drinking water, risk quotients (RQs) were calculated by comparing the concentrations of the target PFASs in samples with the health-based guidelines for PFASs in drinking water issued by different authorities or scholars as follows (Eq. (1)). The average and maximum concentrations were used in the RQs calculation to show the overall and the most serious status of PFAS contamination in drinking water. These were defined as $R Q_{\text {mean }}$ and $R_{\max }$. RQs of PFAS mixtures in drinking water were calculated by summing up the RQs of different compounds as in Eq. (2) [35]. Due to lack of health guidelines and negligible concentrations for certain PFASs, their RQs were neglected:

$$
\begin{aligned}
& \mathrm{RQ}=\frac{C_{\text {PFAS }}}{\mathrm{HBVs}} \\
& \mathrm{RQ}_{\text {mix }}=\sum_{i=1}^{n} \frac{C_{\mathrm{PFAS}_{i}}}{\mathrm{HBV}_{i}}
\end{aligned}
$$

where $C_{\text {PFASs }}$ is the concentration of PFASs, ng/L; HBVs are health-based guideline values of PFASs in drinking water proposed by the different agencies or scholars, $\mathrm{ng} / \mathrm{L}$.

\section{Estimation of daily PFAS intake}

The estimated daily intakes (EDIs) of PFASs via drinking water were calculated as in other studies $[5,36]$ and are based on exposure handbook data following equation:

$$
\mathrm{EDI}=\frac{C_{\mathrm{w}} D_{\mathrm{w}}}{B_{\mathrm{w}}},
$$

where $C_{\mathrm{w}}$ is the concentration of PFASs in drinking water, $\mathrm{ng} / \mathrm{L} ; D_{\mathrm{w}}$ is the daily drinking water volume, $\mathrm{L} / \mathrm{d}$; $B_{\mathrm{w}}$ is body weight, $\mathrm{kg}$.

Since the environmental exposure behavior pattern of Chinese residents to water is significantly different from that of other nations, a significant difference was observed between the daily water consumption per body weight in China $(31 \mathrm{~mL})$ compared to values from the United States $(13 \mathrm{~mL})$, Japan $(11 \mathrm{~mL})$ and South Korea $(24 \mathrm{~mL})$. To better understand the PFAS daily exposure related to drinking water in different Chinese regions and specific age groups, exposure parameters were obtained from the Exposure Factors Handbook of Chinese Population shown in Additional file 1: Table S2 [37]. Total daily PFAS intakes were estimated by dividing EDI by the Relative Source Contribution factor (RSC \%) as in the following equation: 


$$
\mathrm{TDI}=\frac{\mathrm{EDI}}{R S C \%}
$$

\section{Results and discussion}

Levels and mixture profiles of PFASs in Chinese drinking water

We reviewed data for a total of eighteen PFASs in 526 drinking water sampled across 66 cities in China, as summarized in Additional file 1: Table S3. According to the sample distribution, East China (208 samples) and South China (104 samples) were most thoroughly investigated relative to Northeast China (52 samples) and Northwest China (23 samples).

As shown in Fig. 1, the mean $\Sigma$ PFASs in drinking water ranged from $0.1-502.9 \mathrm{ng} / \mathrm{L}$. The highest and lowest concentrations were founded in Zigong and Atushi city, respectively. Zigong (502.9 ng/L), Lianyungang (332.6 ng/L), Changshu (122.4 ng/L), Chengdu (119.4 ng/L), Wuxi (93.6 ng/L), Hangzhou (74.1 ng/L), Nanning (64.1 ng/L), Suzhou (61.3 ng/L), Kunming
$(60.4 \mathrm{ng} / \mathrm{L})$ and Chaohu $(59.9 \mathrm{ng} / \mathrm{L})$ were the top 10 cities with the most PFAS contamination in drinking water. It should be noted that relatively high concentrations of PFASs are present in East China, South China and several cities in Southwest China. ANOVA analysis indicated that the mean $\Sigma$ PFASs values in East China were 2.6 folds higher than in North China. This is mainly due to the intensive industrial activities and high population density in those regions, as reported in another study [38]. High concentrations of $\Sigma$ PFASs in Southwest China were in particularly found in Zigong city, with extreme PFOA concentrations up to $3165 \mathrm{ng} / \mathrm{L}$ near a fluorochemical plant revealed by Fang et al. in [30].

The mixture profiles demonstrated that the PFASs in Chinese drinking water were dominated by the PFOA (0.1-93.0\%), PFBA (0-98.7\%) and PFOS (0-82.7\%) as shown in Fig. 2, with variations across different cities. PFBA was the most abundant compound found in Chengdu, Nanning, Yinchuan, Fuzhou, Zhengzhou, Hohhot, Taiyuan, Guiyang, Xi'an, Kunming, Wuhan, Hefei, Urumqi, Harbin, Xining, Changchun, and Lhasa.

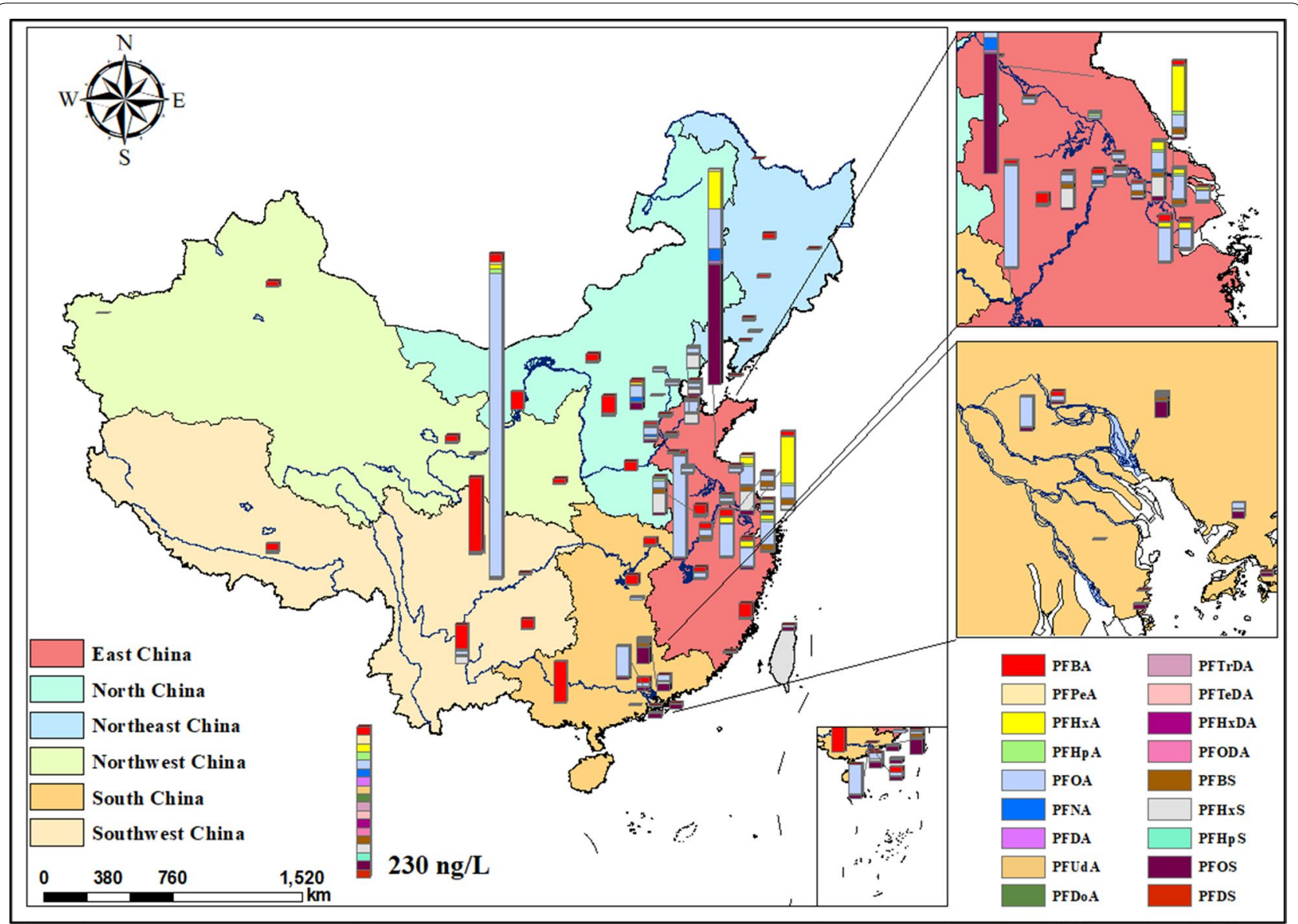

Fig. 1 Spatial distribution of PFASs in drinking water in China 


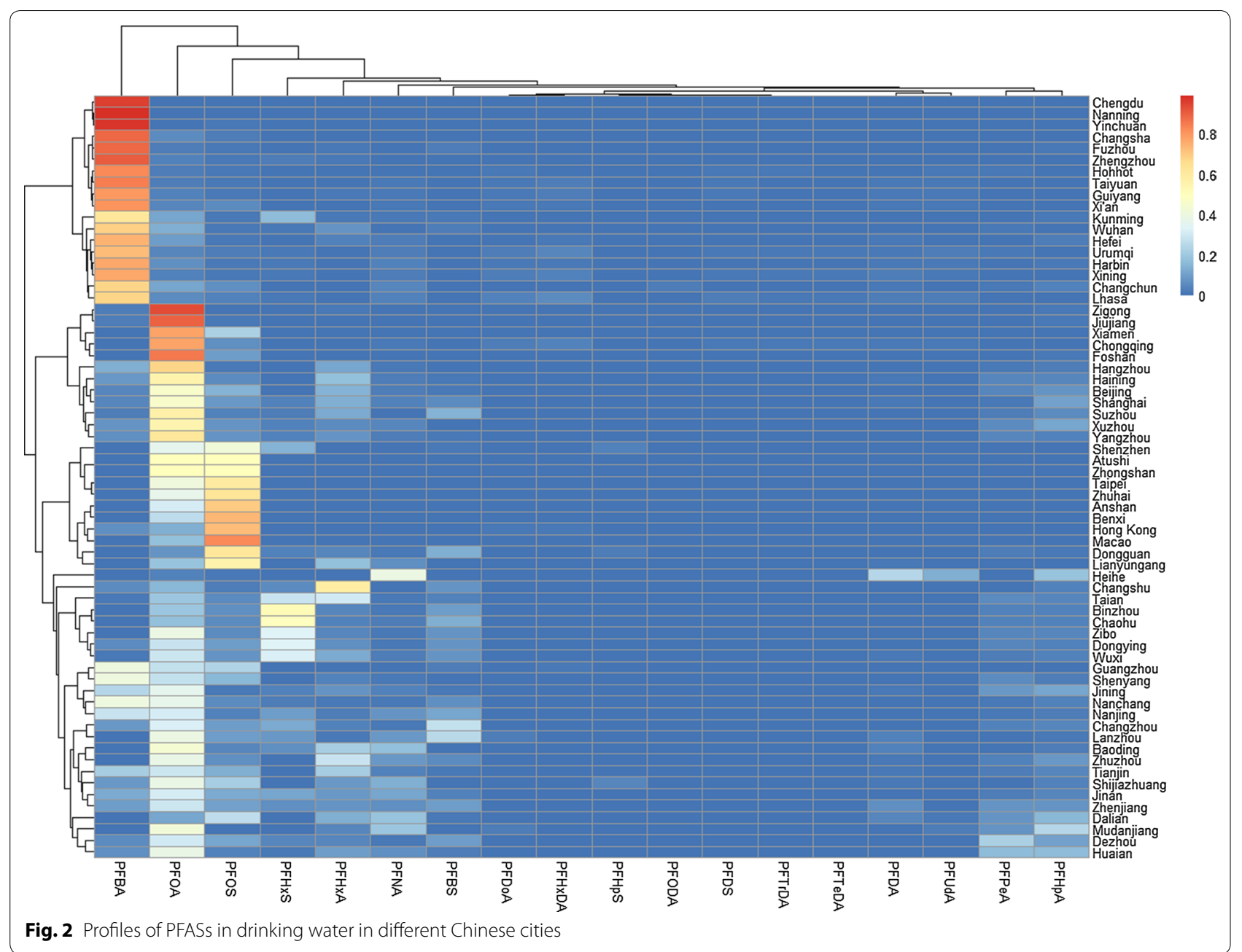

Short-chain PFASs possess limited adsorption to sediments and soils due to their lower Koc, contributing to a relatively high percentage of total PFASs in the aquatic environment [39]. In addition, the removal efficiency of short-chain PFASs during drinking water treatment is relatively limited, even with advanced treatment processes such as granular activated carbon, ion-exchange, and membrane treatment [40]. In some remote areas like Urumqi, Lhasa, and northeastern China, the PFASs in drinking water are thought to mainly originate from longdistance transport. The high percentage of PFBA in these cities is likely due to precursor conversion in the atmosphere [41]. The PFASs in drinking water in other cities were dominated by PFOA and PFOS. The PFOA levels were higher than those of PFOS most likely because of restrictions for several major uses of PFOS since 10 years ago. Extremely high concentrations of PFOA in drinking water were found in Zigong (3165 ng/L) and Jiujiang $(268 \mathrm{ng} / \mathrm{L})$ due to industrial point sources, in particular a fluorochemical plant and multiple industries such as leather, textile and paper manufacturing, which use PFASs in their processes [30, 42]. Previous studies have revealed how the surrounding communities can be contaminated by PFOA emissions from PTFE production facilities, thus resulting in significant human exposure [43]. We investigated the PTFE production capacity of different companies across China in 2018, and the corresponding PFOA concentrations in adjacent cities are summarized in Table 1 . The PFOA concentrations in drinking water in the cities with PTFE production were significantly higher than in those without PTFE production $(\mathrm{p}<0.05)$. The extremely high concentrations of PFOA were mostly observed in provinces with PTFE production such as Sichuan (3165 ng/L), Zhejiang (115.4 ng/L), Shanghai (78 ng/L), Jiangsu (61.4 $\mathrm{ng} / \mathrm{L})$ and Guangdong (53.4 ng/L). These levels were significantly higher than those detected in Korea, Australia and some European countries [3, 44, 45], but comparable to contamination in other cities affected by fluorochemical production such as in Minnesota and New Jersey [24, 46, 
Table 1 Distribution of PTFE production capacity and PFOA concentration in drinking water in nearby cities

\begin{tabular}{llll}
\hline Provinces & Companies & $\begin{array}{l}\text { PTFE production } \\
\text { capacity (10,000 tons) }\end{array}$ & $\begin{array}{l}\text { PFOA concentration in drinking } \\
\text { water in the neighboring city } \\
\text { (ng/L) }\end{array}$ \\
\hline Shandong & Shandong Dongyue Chemical Co., Ltd. & $0-15.89$ \\
Shandong & Luxi Chemical Co., Ltd. & 0.5 & $\mathbf{0 . 1}$ \\
Shandong & Shandong Huafluorin Chemical Co., Ltd. & 0.3 & $18.4-3165$ \\
Sichuan & Zhonghao Chenguang Chemical Research Institute Co., Ltd. & 1.5 & $0-78$ \\
Shanghai & Daikin Fluorine Chemical (China) Co., Ltd. & 1 & $8.1-26.33$ \\
Jiangsu & Sanaifu New Material Technology Co., Ltd. & 1 & $45-268$ \\
Jiangsu & Jiangsu Meilan Chemical Co., Ltd. & 0.6 & $0.13-2.6$ \\
Jiangsu & Changshu Solvay Special Polymer Co., Ltd. & 0.5 & $0.53-115.4$ \\
Jiangxi & Jiangxi Liwen Industrial Co., Ltd. & 0.65 & $1-53.4$ \\
Fujian & Fujian Sannong Chemical Co., Ltd. & 0.6 & $0-4.76$ \\
Zhejiang & Zhejiang Juhua Co., Ltd. & 0.6 & 0.06 \\
Zhejiang & Zhejiang Quzhou Xinju Fluorine Material Co., Ltd. & 0.3 & 0.3 \\
Guangdong & DuPont (China) Co., Ltd. & 0.3 & \\
Liaoning & Liaoning Fuxin Fluorine Chemical Co., Ltd. &
\end{tabular}

47]. The emission source, especially the presence of fluorochemical plants, the major local PFAS industrial users, the use of PFAS-containing firefighting foam [17, 41, 48], and the quality of the drinking water treatment processes and precursor conversion $[48,49]$ can impact the pattern and concentration of PFASs in drinking water. To better inform management and mitigation measures to reduce contamination of PFASs in drinking water, further research is needed to clarify and quantify how these factors impact the specific PFAS mixture profiles observed.

\section{Temporal trend}

Despite their the global phaseout, of PFOS and especially PFOA are still the dominant PFAS species detected in tap water samples across China due to their continued use in China, extreme persistence and long-term release from groundwater reservoirs, and the possible contribution of precursors conversion over time $[48,49]$. In recent years, several companies have announced efforts to research PFOA alternatives. However, the monitoring study in Zigong city revealed the continuous use and production of PFOA by the local company, suggesting that the transition to an alternative is challenging. Thus, better process recovery, emission reduction measures and efficient advanced drinking water processes to control the PFAS contamination in drinking water in China are urgently needed in addition to the search and use of eco-friendly alternatives in the PFAS-producing and using plants which can e.g. degrade in the environment and are not of concern for health.

In addition, a strong increase in short chain PFASs was observed by comparing the samples taken before 2010
[20, 32] with those taken after 2010 [38, 41], indicating that short chain PFASs in Chinese drinking water need more attention and source tracking, followed by efficient control and reduction $[50,51]$. Furthermore, in recent years, some newly identified PFASs were detected in Chinese drinking water, such as FHxSA, PFEtS, F-53B and HFPO-TA $[52,53]$. It is likely that with the further elimination of long-chain PFASs, the production and use of new PFAS alternatives will further increase, leading to their wider occurrence in surface water [54]. Therefore, these PFASs and their precursors in drinking water should receive more attention, monitoring and regulation considering the insufficient assessment of their health effects and the inability of common drinking water treatments to remove these PFASs.

\section{Risk assessment of PFASs in Chinese drinking water}

To further understand the geographical risk distribution of PFASs in Chinese drinking water, risk quotients (RQs) were calculated by comparing average and maximum concentrations with several recent health-based guideline levels, which were denoted as $R Q_{\text {mean }}$ and $R Q_{\max }$. Using the Chinese health advisory values of $85 \mathrm{ng} / \mathrm{L}$ for PFOA and $47 \mathrm{ng} / \mathrm{L}$ for PFOS derived by Zhang et al. [31], the $R_{\text {mean }}$ of sum PFOA and PFOS in 66 cities were in the range of 0.0004-5.54 as illustrated in Fig. 3a. The cities of Zigong, Lianyungang and Jiujiang had the three highest $\mathrm{RQ}_{\text {mean }}: 5.54,4.68$, and 1.89 , respectively. The $\mathrm{RQ}_{\max }$, calculated using the highest detected concentrations, in Zigong exceeded 37, reaching an excessive level. Twenty-four of the 66 cities had $\mathrm{RQ}_{\text {mean }}$ in the range of $0.1-1$, indicating medium health risk from drinking 


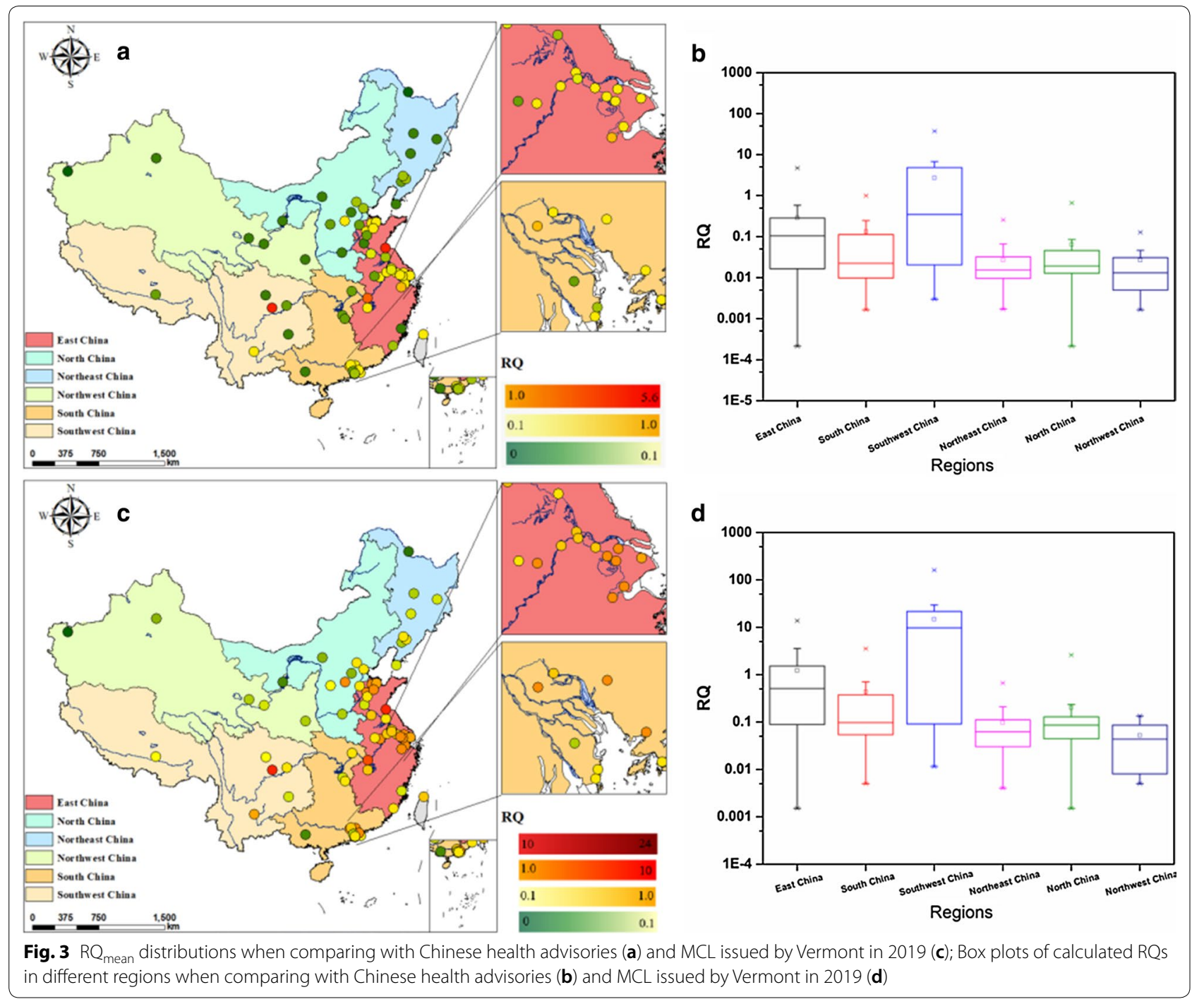

water according to the Chinese national standard [38]. The other thirty-nine cities had negligible risk, with an $\mathrm{RQ}<0.1$. To better understand the overall risk derived from PFASs in different regions, concentrations found in the studies reviewed here were used for the RQ calculation, as shown in Fig. 3b and d. The spatial risk distribution revealed that East China and Southwest China have a significantly higher risk $(p<0.05)$ relative to other regions due to the presence of fluorochemical plants, the high population density, and the intensive paper, textile, and leather industries, as mentioned above.

However, increasingly stricter standards have recently been established in other countries. Comparing with the MCL of $20 \mathrm{ng} / \mathrm{L}$ for PFOA, PFOS, PFHxS, PFHpA, and PFNA individually or combined issued by Vermont in 2019, which is still greater than the recent TWI recommended by EFSA [55], the $R_{\text {mean }}$ of sum PFOA and PFOS in 66 cities were in the range of 0.005-23.94
(Fig. 3c). The $\mathrm{RQ}_{\text {mean }}$ of Zigong, Lianyungang, Jiujiang reached 23.94, 13.46 and 8.12, respectively. Furthermore, high $\mathrm{RQ}_{\text {mean }}(>1)$ were observed in sixteen cities including Wuxi, Hangzhou, Foshan, Chaohu, Suzhou, etc., suggesting that approximately 98.5 million people, based on the population distribution as shown in Additional file 1: Table S4, are at significant risk from exposure to PFASs in drinking water. Considering this standard, twenty-four cities, Yinchuan, Nanning, Atushi and Heihe, etc. have negligible $R_{\text {mean }}$ below 0.1 . In addition, the drinking water in more than $40 \%$ of the studied cities, likely affecting 192.6 million people, exceeded the notification levels of $5.1 \mathrm{ng} / \mathrm{L}$ for PFOA and $6.5 \mathrm{ng} / \mathrm{L}$ for PFOS issued by California in 2019 [56], as summarized in Additional file 1: Table S5. Using the more precautionary limit of $1 \mathrm{ng} / \mathrm{L}$ suggested by Grandjean and Budtz-Jørgensen [26], almost all the cities studied exhibited risk levels $>1$. 
These results indicate that PFASs in drinking water in some of the Chinese city have exceeded the Chinese health advisories and most of the cities have exceeded stringent international guideline levels. Therefore, control and treatment measures are urgently needed.

\section{Daily exposure to PFASs via drinking water for the Chinese population}

We estimated the exposure of the Chinese population to PFASs from drinking water according to the Chinese population exposure parameter manual. To do so, we used the average and maximum concentrations of PFOA/ PFOS widely recognized as posing a health concern with tolerable intake values. Six major districts were used including North China, East China, South China, Northwest China, Northeast China, Southwest China, and the population of each region was divided into 14 age groups shown in Additional file 1: Table S2. The EDI $\mathrm{I}_{\text {mean }}$ and $\mathrm{EDI}_{\max }$ of adults in the aforementioned highly contaminated cities were significantly higher than in other studies $[57,58]$.

In addition, total daily intakes (TDI) of PFASs were estimated by applying a conservative Relative Source Contribution factor (RSC) equal to $10 \%$, assuming that $10 \%$ of the PFAS exposure comes from direct drinking water consumption [59]. Similarly, the average and maximum PFAS concentration were used to calculate $\mathrm{TDI}_{\text {mean }}$ and TDI $\mathrm{max}_{\text {. The estimated TDI }}$ mean of PFOA and PFOS for adults ranged from 0 to $121.7 \mathrm{ng} / \mathrm{kg} /$ day and $0-61.43 \mathrm{ng} / \mathrm{kg} /$ day across China, respectively. The estimated TDI mean $_{\text {of highly PFOA/PFOS contaminated }}$ drinking water is compiled in Fig. 4. Infants and toddlers from 9 months to 2 years of age had the highest risk of PFOA/PFOS exposure due to their relatively higher water consumption per unit weight. This is in line with other studies [5, 35]. Some discriminations regarding to the risk variation trends among specific age groups were also found, since the varied population exposure parameters were used [35]. Moreover, we used toxicity values showing no adverse effects for long-term human exposure, such as Tolerable Daily/Weekly Intake and Reference dose values, to assess the PFAS exposure risk for the Chinese population.

The Agency for Toxic Substances and Disease Registry (ATSDR) proposed updated Tolerable Daily Intake values of $2 \mathrm{ng} / \mathrm{kg} /$ day for PFOS and $3 \mathrm{ng} / \mathrm{kg} /$ day for PFOA in 2018 [60]. Furthermore, the European Food Safety Authority (EFSA) derived the slightly lower Tolerable Weekly Intake values (TWIs) of $6 \mathrm{ng} / \mathrm{kg} /$ week for PFOA and $13 \mathrm{ng} / \mathrm{kg} /$ week for PFOS in 2018 [61]. In 2020, the EFSA published a scientific opinion establishing a new tolerable weekly intake of $4.4 \mathrm{ng} / \mathrm{kg}$ for the sum of PFOA, PFOS, PFHxS and PFNA, which lowers the EFSA 2018 regulation in practice [55]. These Tolerable Daily/Weekly Intake values are significantly lower than the healthbased guidance values for tolerable intake previously issued by other international bodies, as summarized in Table 2.

Compared with these recent standards, the total PFOA intake for all age groups exceeds $3 \mathrm{ng} / \mathrm{kg} /$ day in Zigong, Jiujiang, Lianyungang, Foshan, Suzhou, Wuxi, Haining, Changshu, Shijiazhuang, Zibo and Shanghai. These high exposures are mostly concentrated in East China given the high PFOA contamination and daily intake from water. Concerns about human exposure risk in these cities deserve more attention. As for PFOS, the TDI $I_{\text {mean }}$ for adults in Lianyungang, Dongguan, Shenzhen, Shijiazhuang, Wuxi, Macao, HongKong, Taipei, Foshan, Guangzhou, Jinan, Chaohu, Changshu ranged from 1.27 to $61.44 \mathrm{ng} / \mathrm{kg} /$ day, while ranging from 3.54 to $171.28 \mathrm{ng} / \mathrm{kg} /$ day for infants between 9 months and 1 year, significantly exceeding the tolerable daily/weekly intake values of $2 \mathrm{ng} / \mathrm{kg} /$ day and $13 \mathrm{ng} / \mathrm{kg} /$ week, respectively. It should be noted that the $\mathrm{TDI}_{\max }$ in Zigong city ranged from 822.9 to $2975.1 \mathrm{ng} / \mathrm{kg} /$ day for different age groups, exceeding the tolerable daily intake value issued by ATSDR 274.3 times and 991.7 times, respectively. This highlights that the human exposure via drinking water daily intake in highly contaminated regions such as Zigong, Lianyungang and Jiujiang is excessive considering these science-based tolerable intake values, with a particular high risk for infants.

\section{Conclusion}

As the most populated country with a large production and use of PFASs, including PTFE, China is at high risk of PFAS exposure for millions of its residents, as already documented in the United States [62]. Therefore, this study reviewed and assessed all available PFAS drinking water data in China and compared the levels with recently lowered drinking water guidelines and tolerable daily/weekly intake values from other industrial countries. This is the first comprehensive study reviewing the PFAS levels in Chinese drinking water and assessing the associated heath risk. PFASs have been frequently detected in drinking water across China, sometimes at high concentrations [63]. The results showed that PFOA, PFOS and PFBA were the dominant PFAS species in Chinese drinking water. East China and Southwest China had higher concentrations of PFASs compared with other regions. The cities of Zigong, Lianyungang, and Jiujiang, with point sources like fluorochemical plants and multiple PFAS-using industries such as leather, textile and paper, etc., where found to generally pose a higher risk to humans from PFAS exposure via drinking water, above Chinese and 


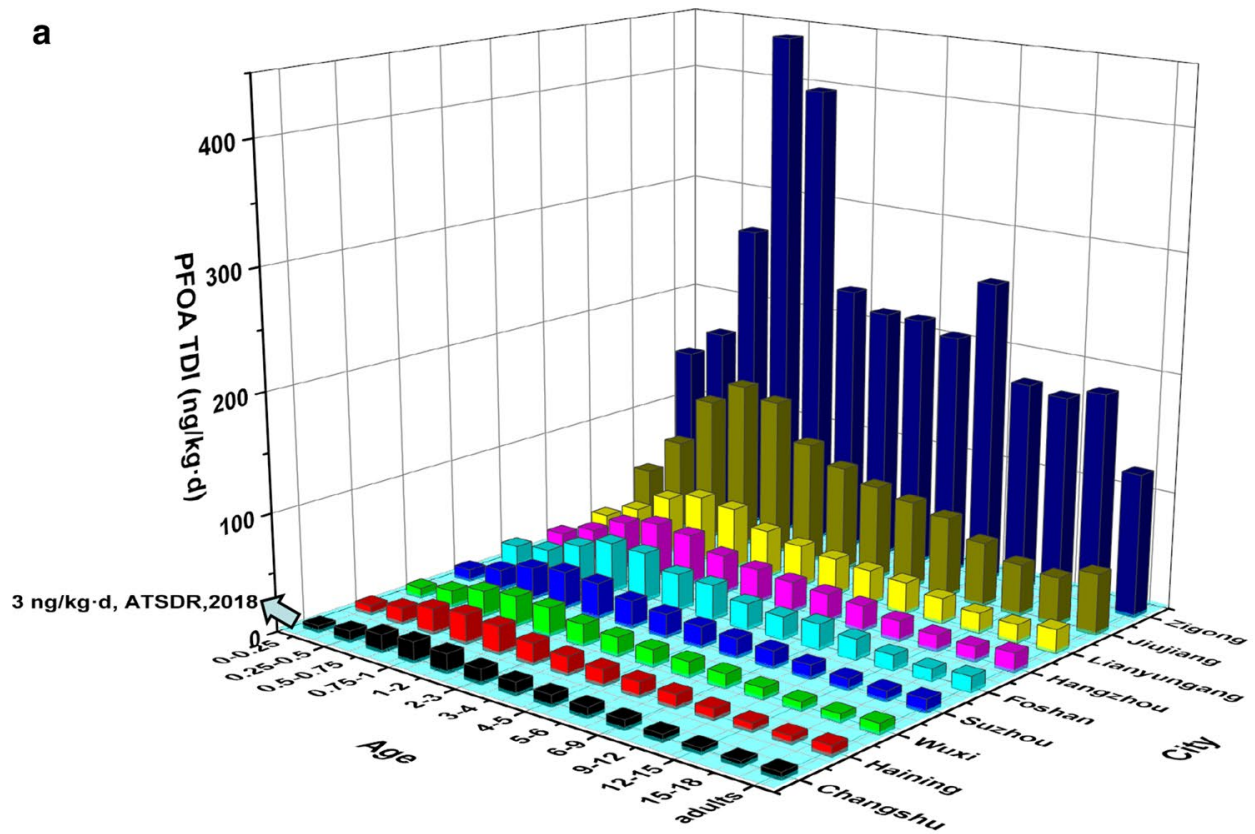

b

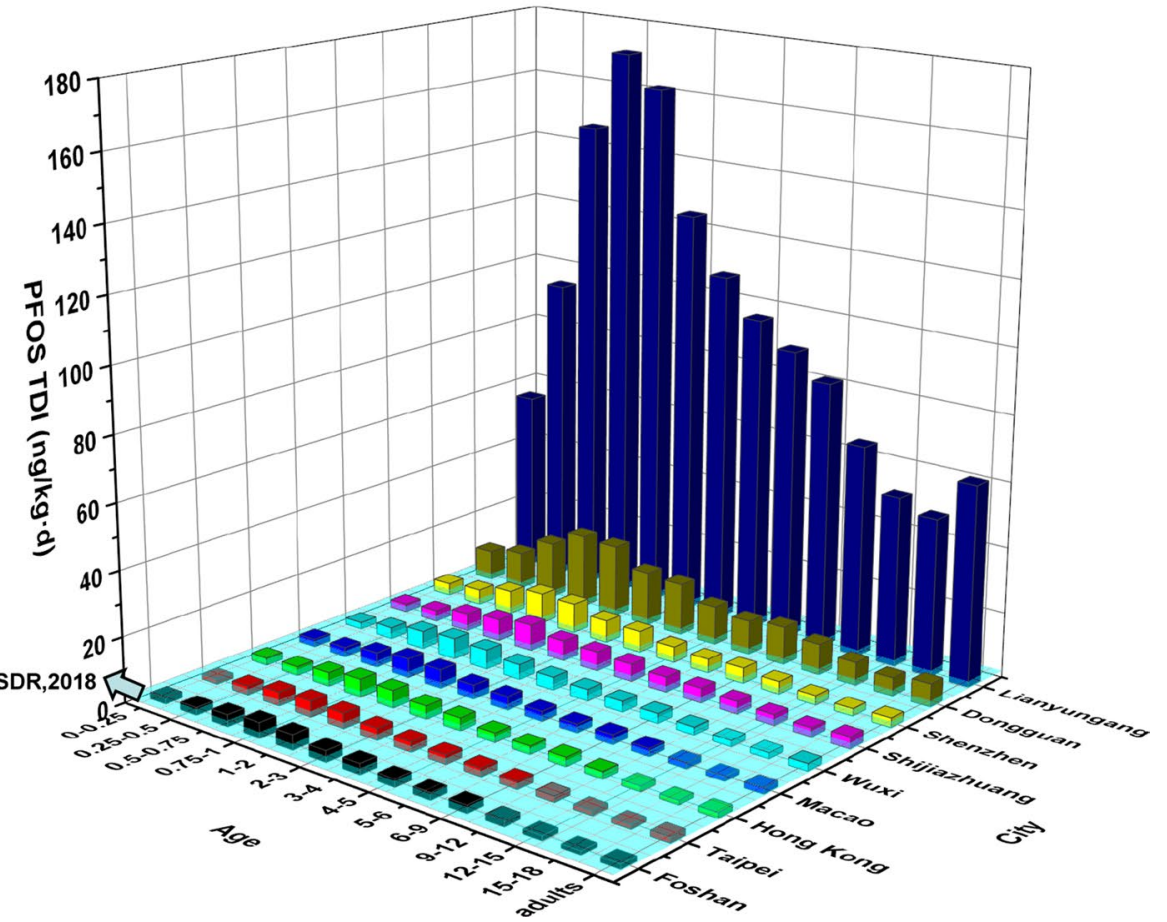

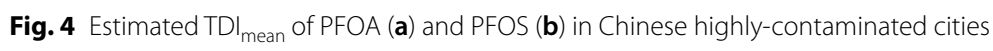

international risk limits. These and other 13 of the total 66 cities with available PFAS data exhibited high RQs (>1) when comparing with the MCL issued by Vermont in 2019. The total daily intake of PFOA and PFOS also exceeded the Tolerable Daily/Weekly Intake values issued by ATSDR and EFSA in the aforementioned regions, especially for infants and toddlers 9 months to 2 years. Therefore, the PFAS contamination of drinking water in highly polluted areas needs urgent attention and point source control. More than $20 \%$ of the studied 
Table 2 Tolerable intake values issued by different agencies ( $\mathrm{ng} / \mathrm{kg}$ body weight)

\begin{tabular}{llll}
\hline Agencies & PFOS & PFOA & Type \\
\hline Europe (EFSA, 2008) & 150 & 1500 & Tolerable Daily Intake \\
Europe (EFSA, 2018) & 13 & 6 & Tolerable Weekly Intake \\
Europe (EFSA, 2020) & $4.4^{*}$ & $4.4^{*}$ & Tolerable Weekly Intake \\
U.S. (EPA, 2016) & 20 & 20 & Reference Dose \\
U.S. (ATSDR, 2018) & 2 & 3 & Tolerable Daily Intake \\
Netherlands(RIVM, 2016) & - & 12.5 & Tolerable Daily Intake \\
Australia (enHealth, 2016) & 150 & 1500 & Tolerable Daily Intake \\
Australia (FSANZ, 2017) & 20 & 160 & Tolerable Daily Intake \\
\hline
\end{tabular}

* Means the sum of PFOA, PFOS, PFHxS and PFNA

cities, likely affecting 98.5 million people, had drinking water with PFAS levels above stringent and sciencebased international limits, which demonstrates a need for urgent attention and countermeasures.

However, this study also has some uncertainties. Given the wide variability in the number of measurements in different regions, the analytical methods used in different studies, the sampling dates, toxicity studies considered, etc., all of which influence the calculated concentrations and risk assessment, we included all the published data points in Additional file 1: Table S6 to inform future studies. More research is needed to understand the possible health effect associated with long-term exposure in hot spots, especially in areas with PFASs-related manufacturing plants, and in particular PTFE manufacturing. Moreover, better monitoring of PFASs in drinking water is needed in remote areas, to provide a full overview of PFAS contamination in Chinese drinking water [64].

Finally, the current risk assessment of PFASs in China should be re-evaluated in light of the most recent toxicological studies, to determine if the guideline values need to be lowered as recently done in Europe and the United States. This is critical to have the best national basis for risk assessment and a strong driver for countermeasures.

\section{Supplementary information}

Supplementary information accompanies this paper at https://doi. org/10.1186/s12302-020-00425-3.

Additional file 1: Table S1. Guidelines for PFASs in drinking water issued by different countries and U.S. states. Table S2. Chinese population exposure parameters via drinking water. Table S3. Calculated mean concentrations of PFASs in drinking water in Chinese cities (ng/L). Table S4. Location and population information of the studied cities. Table S5. Calculated RQs via comparing with the different guidelines. Table S6. Concentrations of PFASs in Chinese drinking water in the published literatures (ng/L) and reference list by literature survey.

Abbreviations

PFASs: Perfluoroalkyl and polyfluoroalkyl substances; EFSA: European Food Safety Authority; ATSDR: Agency for Toxic Substances and Disease Registry;
TDI: Total daily intake; RQ: Risk quotient; PFBA: Perfluorobutanoic acid; PFOS: Perfluorooctane sulfonate; PFOA: Perfluorooctanoic acid; PFHxS: Perfluorohexane sulfonate; PFHXA: Perfluorohexanoic acid; PFNA: Perfluorononanoic acid; PFBS: Perfluorobutane sulfonate; PFDoA: Perfluorododecanoic acid; PFHXDA: Perfluorohexadecanoic acid; PFHpS: Perfluoroheptane sulfonate; PFODA: Perfluorooctadecanoic acid; PFDS: Perfluorodecane sulfonate; PFTrDA: Perfluorotridecanoic acid; PFTeDA: Perfluorotetradecanoic acid; PFDA: Perfluorodecanoic acid; PFUdA: Perfluoroundecanoic acid; PFPeA: Perfluoropentanoic acid; PFHpA: Perfluoroheptanoic acid.

\section{Acknowledgements}

The authors would like to acknowledge funding from the Major Science and Technology Program for Water Pollution Control and Treatment in China and Simona Balan for her valuable comments and native editing.

\section{Authors' contributions}

LL, YQ: Investigation, validation, writing—Original draft preparation. JH: Conceptualization, methodology, writing — review \& editing. RW: Writing, global context, review \& editing. All authors read and approved the final manuscript.

\section{Funding}

This work was financially supported by the Major Science and Technology Program for Water Pollution Control and Treatment in China (Nos. 2017ZX07202001 and 2017ZX07202-004).

Availability of data and materials

Not applicable.

Ethics approval and consent to participate

Not applicable.

\section{Consent for publication}

Not applicable.

\section{Competing interests}

The authors declare no competing financial interest.

\section{Author details}

${ }^{1}$ State Key Joint Laboratory of Environmental Simulation and Pollution Control (SKLESPC), Beijing Key Laboratory for Emerging Organic Contaminants Control (BKLEOC), Beijing Laboratory for Environmental Frontier Technologies (BLEFT), School of Environment, Tsinghua University, Beijing 100084, China. ${ }^{2}$ POPs Environmental Consulting, Lindenfirststr. 23, 73527 Schwäbisch Gmünd, Germany.

Received: 17 July 2020 Accepted: 21 October 2020

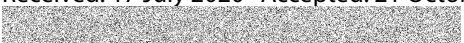

References

1. Jin YH, Liu W, Sato I, Nakayama SF, Sasaki K, Saito N et al (2009) PFOS and PFOA in environmental and tap water in China. Chemosphere 77:605-611. https://doi.org/10.1016/j.chemosphere.2009.08.058

2. Prevedouros K, Cousins IT, Buck RC, Korzeniowski SH (2006) Sources, fate and transport of perfluorocarboxylates. Environ Sci Technol 40:32-44. https://doi.org/10.1021/es0512475

3. Thompson J, Eaglesham G, Mueller J (2011) Concentrations of PFOS, PFOA and other perfluorinated alkyl acids in Australian drinking water. Chemosphere 83:1320-1325. https://doi.org/10.1016/j.chemospher e.2011.04.017

4. Kunacheva C, Fujii S, Tanaka S, Seneviratne ST, Lien NP, Nozoe M et al (2012) Worldwide surveys of perfluorooctane sulfonate (PFOS) and perfluorooctanoic acid (PFOA) in water environment in recent years. Water Sci Technol 66:2764-2771. https://doi.org/10.2166/wst.2012.518

5. Ao J, Yuan T, Xia H, Ma Y, Shen Z, Shi R et al (2019) Characteristic and human exposure risk assessment of per- and polyfluoroalkyl substances: a study based on indoor dust and drinking water in China. Environ Pollut 254:112873. https://doi.org/10.1016/j.envpol.2019.07.041

6. Wang T, Wang P, Meng J, Liu S, Lu Y, Khim JS et al (2015) A review of sources, multimedia distribution and health risks of perfluoroalkyl acids 
(PFAAs) in China. Chemosphere 129:87-99. https://doi.org/10.1016/j. chemosphere.2014.09.021

7. Banjabi AA, Li AJ, Kumosani TA, Yousef JM, Kannan K (2020) Serum concentrations of perfluoroalkyl substances and their association with osteoporosis in a population in Jeddah, Saudi Arabia. Environ Res 187:109676. https://doi.org/10.1016/j.envres.2020.109676

8. Dauchy X (2019) Per- and polyfluoroalkyl substances (PFASs) in drinking water: current state of the science. Curr Opin Environ Health 7:8-12. https://doi.org/10.1016/j.coesh.2018.07.004

9. Lorber M, Egeghy PP (2011) Simple intake and pharmacokinetic modeling to characterize exposure of Americans to perfluoroctanoic acid, PFOA. Environ Sci Technol 45:8006-8014. https://doi.org/10.1021/ es103718h

10. Sunderland EM, Hu XC, Dassuncao C, Tokranov AK, Wagner CC, Allen JG (2019) A review of the pathways of human exposure to poly-and perfluoroalkyl substances (PFASs) and present understanding of health effects. J Expo Sci Environ Epidemiol 29:131-147. https://doi. org/10.1038/s41370-018-0094-1

11. LuY, Luo B, Li J, Dai JY (2016) Perfluorooctanoic acid disrupts the blood-testis barrier and activates the TNFa/p38 MAPK signaling pathway in vivo and in vitro. Arch Toxicol 90(4):971-983. https://doi. org/10.1007/s00204-015-1492-y

12. Liu WX, Wu JY, He W, Xu FL (2019) A review on perfluoroalkyl acids studies: environmental behaviors, toxic effects, and ecological and health risks. Ecosyst Health Sustain 5(1):1-19. https://doi. org/10.1080/20964129.2018.1558031

13. Steenland K, Fletcher T, Savitz DA (2010) Epidemiologic evidence on the health effects of perfluorooctanoic acid (PFOA). Environ Health Perspect 118:1100-1108. https://doi.org/10.1289/ehp.0901827

14. US EPA (2016) Drinking water health advisories for PFOA and PFOS. https://www.epa.gov/ground-water-and-drinking-water/drinkingwater-health-advisories-pfoa-and-pfos

15. Stockholm Convention (2009) The New POPs Under the Stockholm Convention. http://chm.pops.int/Programmes/NewPOPs/The9newPOP s/tabid/672/language/en-US/Default.aspx

16. Stockholm Convention (2019) POPRC Recommendations for listing Chemicals. http://www.pops.int/TheConvention/ThePOPs/ChemicalsP roposedforListing/tabid/2510/Default.aspx

17. $H u X C$, Andrews DQ, Lindstrom AB, Bruton TA, Schaider LA, Grandjean P et al (2016) Detection of Poly- and Perfluoroalkyl Substances (PFASs) in U.S. Drinking Water Linked to Industrial Sites, Military Fire Training Areas, and Wastewater Treatment Plants. Environ Sci Technol Lett 3:344-350. https://doi.org/10.1021/acs.estlett.6b00260

18. Essumang DK, Eshun A, Hogarh JN, Bentum JK, Adjei JK, Negishi J et al (2017) Perfluoroalkyl acids (PFAAs) in the Pra and Kakum River basins and associated tap water in Ghana. Sci Total Environ 579:729-735. https://doi.org/10.1016/j.scitotenv.2016.11.035

19. Unlu Endirlik B, Bakir E, Bosgelmez II, Eken A, Narin I, Gurbay A (2019) Assessment of perfluoroalkyl substances levels in tap and bottled water samples from Turkey. Chemosphere 235:1162-1171. https://doi. org/10.1016/j.chemosphere.2019.06.228

20. Qiu Y, Jing H, Shi H (2010) Perfluorocarboxylic acids (PFCAs) and perfluoroalkyl sulfonates (PFASs) in surface and tap water around Lake Taihu in China. Front Environ Sci Eng 4:301-310. https://doi. org/10.1007/s11783-010-0236-8

21. Takagi S, Adachi F, Miyano K, Koizumi Y, Tanaka H, Mimura M et al (2008) Perfluorooctanesulfonate and perfluorooctanoate in raw and treated tap water from Osaka, Japan. Chemosphere 72:1409-1412. https://doi.org/10.1016/j.chemosphere.2008.05.034

22. Cordner A, De La Rosa VY, Schaider LA, Rudel RA, Richter L, Brown $P$ (2019) Guideline levels for PFOA and PFOS in drinking water: the role of scientific uncertainty, risk assessment decisions, and social factors. J Expo Sci Environ Epidemiol 29:157-171. https://doi.org/10.1038/s4137 0-018-0099-9

23. Soechtig S, Seifert J (2018) The devil we know. https://thedevilweknow. $\mathrm{com} /$

24. Oliaei F, Kriens D, Weber R, Watson A (2013) PFOS and PFC releases and associated pollution from a PFC production plant in Minnesota (USA). Environ Sci Pollut Res 20:1977-1992. https://doi.org/10.1007/s1135 6-012-1275-4
25. https://dec.vermont.gov/water/drinking-water/water-quality-monit oring/pfas

26. Grandjean P, Budtz-Jørgensen E (2013) Immunotoxicity of perfluorinated alkylates: calculation of benchmark doses based on serum concentrations in children. Environ Health 12:35. https://doi. org/10.1186/1476-069X-12-35

27. Chen C, LuY, Zhang X, Geng J, Wang T, Shi Y et al (2009) A review of spatial and temporal assessment of PFOS and PFOA contamination in China. Chem Ecol 25:163-177. https://doi.org/10.1080/02757540902918321

28. Song X, Vestergren R, Shi Y, Huang J, Cai Y (2018) Emissions, Transport, and Fate of Emerging Per- and Polyfluoroalkyl Substances from One of the Major Fluoropolymer Manufacturing Facilities in China. Environ Sci Technol 52:9694-9703. https://doi.org/10.1021/acs.est.7b06657

29. Lu Z, Lu R, Zheng H, Yan J, Song L, Wang J et al (2018) Risk exposure assessment of per- and polyfluoroalkyl substances (PFASs) in drinking water and atmosphere in central eastern China. Environ Sci Pollut Res Int 25:9311-9320. https://doi.org/10.1007/s11356-017-0950-x

30. Fang S, Sha B, Yin H, Bian Y, Yuan B, Cousins IT (2020) Environment occurrence of perfluoroalkyl acids and associated human health risks near a major fluorochemical manufacturing park in southwest of China. J Hazard Mater 396:122617. https://doi.org/10.1016/j.jhazmat.2020.122617

31. Zhang S, Kang Q, Peng H, Ding M, Zhao F, Zhou Y et al (2019) Relationship between perfluorooctanoate and perfluorooctane sulfonate blood concentrations in the general population and routine drinking water exposure. Environ Int 126:54-60. https://doi.org/10.1016/j.envint.2019.02.009

32. Mak YL, Taniyasu S, Yeung LWY, Lu G, Jin L, Yang Y et al (2009) Perfluorinated compounds in tap water from China and several other countries. Environ Sci Technol 43:4824-4829. https://doi.org/10.1021/es900637a

33. GB5749-2006. Sanitary standard for drinking water in China [S]. http:// www.chinacdc.cn/jdydc/200701/P02007011832214491992266574920 06497.pdf

34. Lu GH, Gai N, Zhang P, Piao HT, Chen S, Wang XC et al (2017) Perfluoroalkyl acids in surface waters and tapwater in the Qiantang River watershed-Influences from paper, textile, and leather industries. Chemosphere 185:610-617. https://doi.org/10.1016/j.chemosphere.2017.06.139

35. Thomaidi VS, Tsahouridou A, Matsoukas C, Stasinakis AS, Petreas M, Kalantzi OI (2020) Risk assessment of PFASs in drinking water using a probabilistic risk quotient methodology. Sci Total Environ 712:136485. https://doi.org/10.1016/j.scitotenv.2019.136485

36. Zhang T, Sun H, Lin Y, Wang L, Zhang X, Liu Y et al (2011) Perfluorinated compounds in human blood, water, edible freshwater fish, and seafood in China: daily intake and regional differences in human exposures. J Agric Food Chem 59:11168-11176. https://doi.org/10.1021/jf2007216

37. Duan XL et al (2013) Exposure factors handbook of Chinese population [M]. China Environmental Press, Beijing

38. Chen R, Li G, Yu Y, Ma X, Zhuang Y, Tao H et al (2019) Occurrence and transport behaviors of perfluoroalkyl acids in drinking water distribution systems. Sci Total Environ 697:134162. https://doi.org/10.1016/j.scito tenv.2019.134162

39. Zhao P, Xia X, Dong J, Xia N, Jiang X, Li Y et al (2016) Short- and long-chain perfluoroalkyl substances in the water, suspended particulate matter, and surface sediment of a turbid river. Sci Total Environ 568:57-65. https://doi. org/10.1016/j.scitotenv.2016.05.221

40. Bertanza G, Capoferri GU, Carmagnani M, Icarelli F, Sorlini S, Pedrazzani R (2020) Long-term investigation on the removal of perfluoroalkyl substances in a full-scale drinking water treatment plant in the Veneto Region, Italy. Sci Total Environ 734:139154. https://doi.org/10.1016/j.scito tenv.2020.139154

41. LiY, Li J, Zhang L, Huang Z, Liu Y, Wu N et al (2019) Perfluoroalkyl acids in drinking water of China in 2017: distribution characteristics, influencing factors and potential risks. Environ Int 123:87-95. https://doi. org/10.1016/j.envint.2018.11.036

42. Tan KY, Lu GH, Yuan X, Zheng Y, Shao PW, Zhao YR et al (2018) Perfluoroalkyl Substances in Water from the Yangtze River and Its Tributaries at the Dividing Point Between the Middle and Lower Reaches. Bull Environ Contam Toxicol 101(5):598-603. https://doi.org/10.1007/s00128-018-2444-z

43. Bao J, Liu W, Liu L, Jin Y, Dai J, Ran X et al (2011) Perfluorinated Compounds in the Environment and the Blood of Residents Living near Fluorochemical Plants in Fuxin, China. Environ Sci Technol 45:8075-8080. https://doi.org/10.1021/es102610x 
44. Ericson I, Domingo JL, Nadal M, Bigas E, Llebaria X, van Bavel B et al (2009) Levels of perfluorinated chemicals in municipal drinking water from Catalonia, Spain: public health implications. Arch Environ Contam Toxicol 57:631-638. https://doi.org/10.1007/s00244-009-9375-y

45. Kim S-K, Kho YL, Shoeib M, Kim K-S, Kim K-R, Park J-E et al (2011) Occurrence of perfluorooctanoate and perfluorooctanesulfonate in the Korean water system: implication to water intake exposure. Environ Pollu 159:1167-1173. https://doi.org/10.1016/j.envpol.2011.02.004

46. Post GB, Louis JB, Cooper KR, Boros-Russo BJ, Lippincott RL (2009) Occurrence and Potential Significance of Perfluorooctanoic Acid (PFOA) Detected in New Jersey Public Drinking Water Systems. Environ Sci Technol 43:4547-4554. https://doi.org/10.1021/es900301s

47. Steenland K, Jin C, MacNeil J, Lally C, Ducatman A, Vieira V et al (2009) Predictors of PFOA Levels in a Community Surrounding a Chemical Plant. Environ Health Perspect 117:1083-1088. https://doi.org/10.1289/ ehp.0800294

48. Mumtaz M, Bao Y, Liu L, Huang J, Cagnetta G, Yu G (2019) Per- and polyfluoroalkyl substances in representative fluorocarbon surfactants used in Chinese film-forming foams: levels, profile shift, and environmental implications (vol 6, pg 259, 2019). Environ Sci Technol Lett 6:430. https:// doi.org/10.1021/acs.estlett.9b00330

49. Mumtaz M, Bao Y, Li W, Kong L, Huang J, Yu G (2019) Screening of textile finishing agents available on the Chinese market: an important source of per- and polyfluoroalkyl substances to the environment. Front Environ Sci Eng. https://doi.org/10.1007/s11783-019-1145-0

50. Guardian MGE, Boongaling EG, Bernardo-Boongaling VRR, Gamonchuang J, Boontongto T, Burakham R et al (2020) Prevalence of per- and polyfluoroalkyl substances (PFASs) in drinking and source water from two Asian countries. Chemosphere 256:127115. https://doi.org/10.1016/j. chemosphere.2020.127115

51. Park H, Choo G, Kim H, Oh JE (2018) Evaluation of the current contamination status of PFASs and OPFRs in South Korean tap water associated with its origin. Sci Total Environ 634:1505-1512. https://doi.org/10.1016/j.scito tenv.2018.04.068

52. Qu Y, Jiang X, Cagnetta G, Liu L, Bao Y, Li W et al (2019) Poly- and perfluoroalkyl substances in a drinking water treatment plant in the Yangtze River Delta of China: temporal trend, removal and human health risk. Sci Total Environ. https://doi.org/10.1016/j.scitotenv.2019.133949

53. Kabore HA, Vo Duy S, Munoz G, Meite L, Desrosiers M, Liu J et al (2018) Worldwide drinking water occurrence and levels of newly-identified perfluoroalkyl and polyfluoroalkyl substances. Sci Total Environ 616-617:1089-1100. https://doi.org/10.1016/j.scitotenv.2017.10.210

54. Zhou J, Li Z, Guo X, Li Y, Wu Z, Zhu L (2019) Evidences for replacing legacy per- and polyfluoroalkyl substances with emerging ones in Fen and Wei
River basins in central and western China. J Hazard Mater 377:78-87. https://doi.org/10.1016/j.jhazmat.2019.05.050

55. EFSA (2020) Risk to human health related to the presence of perfluoroalkyl substances in food. EFSA J 18(9):e06223. https://doi.org/10.2903/j. efsa.2020.6223

56. https://www.ecos.org/news-and-updates/california-lowers-pfas-notificati on-levels-initiates-regulatory-process/

57. Zhou W, Zhao S, Tong C, Chen L, Yu X, Yuan T et al (2019) Dietary intake, drinking water ingestion and plasma perfluoroalkyl substances concentration in reproductive aged Chinese women. Environ Int 127:487-494. https://doi.org/10.1016/j.envint.2019.03.075

58. Poothong S, Papadopoulou E, Padilla-Sanchez JA, Thomsen C, Haug LS (2020) Multiple pathways of human exposure to poly- and perfluoroalkyl substances (PFASs): from external exposure to human blood. Environ Int 134:105244. https://doi.org/10.1016/j.envint.2019.105244

59. NHMRC N (2011) Australian drinking water guidelines paper 6 national water quality management strategy. National Health and Medical Research Council, National Resource Management Ministerial Council, Commonwealth of Australia, Canberra, pp 5-7

60. Agency for Toxic Substances and Disease Registry (ATSDR) (2018) Toxicological Profile for Perfluoroalkyls: Draft for Public Comment; https://www. atsdr.cdc.gov/toxprofiles/tp200.pdf

61. EFSA (2018) Panel on Contaminants in the Food Chain (CONTAM), risk to human health related to the presence of perfluorooctane sulfonic acid and perfluorooctanoic acid in food. EFSA J 16(12):e05194. https://doi. org/10.2903/j.efsa.2018.5194

62. Environmental Working Group (2018) Report: Up to 110 Million Americans Could Have PFAS-Contaminated Drinking Water - PFAS Tap Water Data Was Funded By Taxpayers But Kept Secret. https://www.ewg.org/ research/report-110-million-americans-could-have-pfas-contaminateddrinking-water

63. Tan KY, Lu GH, Piao HT, Chen S, Jiao XC, Gai N et al (2017) Current contamination status of perfluoroalkyl substances in tapwater from 17 cities in the Eastern China and their correlations with surface waters. Bull Environ Contam Toxicol 99:224-231

64. Domingo JL, Nadal M (2019) Human exposure to per- and polyfluoroalkyl substances (PFAS) through drinking water: a review of the recent scientific literature. Environ Res 177:108648. https://doi.org/10.1016/j.envre s.2019.108648

\section{Publisher's Note}

Springer Nature remains neutral with regard to jurisdictional claims in published maps and institutional affiliations.

\section{Submit your manuscript to a SpringerOpen ${ }^{\circ}$ journal and benefit from:}

- Convenient online submission

- Rigorous peer review

- Open access: articles freely available online

- High visibility within the field

- Retaining the copyright to your article

Submit your next manuscript at $\boldsymbol{\nabla}$ springeropen.com 